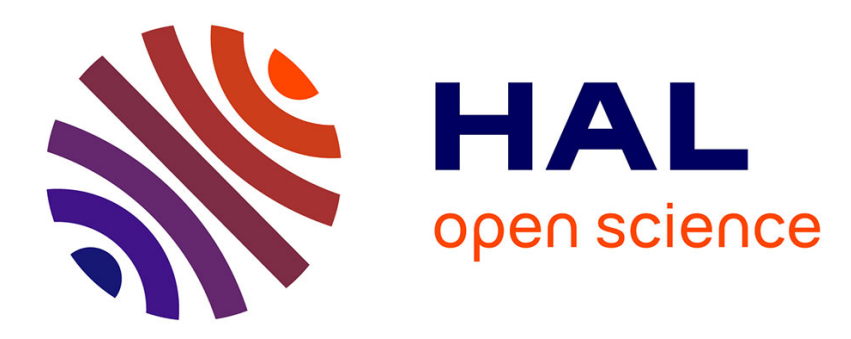

\title{
A renormalization group analysis of ternary polymer solutions
}

\author{
L. Schäfer, Ch. Kappeler
}

\section{To cite this version:}

L. Schäfer, Ch. Kappeler. A renormalization group analysis of ternary polymer solutions. Journal de Physique, 1985, 46 (11), pp.1853-1864. 10.1051/jphys:0198500460110185300 . jpa-00210136

\section{HAL Id: jpa-00210136 https://hal.science/jpa-00210136}

Submitted on 1 Jan 1985

HAL is a multi-disciplinary open access archive for the deposit and dissemination of scientific research documents, whether they are published or not. The documents may come from teaching and research institutions in France or abroad, or from public or private research centers.
L'archive ouverte pluridisciplinaire HAL, est destinée au dépôt et à la diffusion de documents scientifiques de niveau recherche, publiés ou non, émanant des établissements d'enseignement et de recherche français ou étrangers, des laboratoires publics ou privés. 
Classification

Physics Abstracts

$36.20-64.60$

\title{
A renormalization group analysis of ternary polymer solutions
}

\author{
L. Schäfer \\ Fachbereich Physik der Universität Essen 4300 Essen, F.R.G. \\ and Ch. Kappeler \\ Institut für theoretische Physik der Universität Hannover, 3000 Hannover, F.R.G.
}

(Reçu le 8 mars 1985, accepté sous sa forme définitive le 17 juin 1985)

\begin{abstract}
Résumé. - Nous calculons les trajectoires du groupe de renormalisation à l'ordre d'une boucle dans le cas d'une solution comportant deux espèces de polymères dans un solvant commun. Ces trajectoires pourraient être observées par la mesure des rapports d'interpénétration, et nous discutons les fonctions de crossover pour ces quantités à l'ordre d'une boucle. Le flot du groupe de renormalisation détermine également le diagramme de phase du système et nous présentons une analyse détaillée de la courbe spinodale limitant la région de stabilité de la solution homogène. Déjà à l'approximation en arbres, la théorie renormalisée conduit à des résultats significativement différents à la fois de la théorie de Flory-Huggins ou de simples considérations de lois d'échelle.
\end{abstract}

\begin{abstract}
We calculate to the order of one loop the renormalization group trajectories for a solution containing two polymer species in a common solvent. These trajectories could be observed by measuring the interpenetration ratios, and we discuss crossover functions for these quantities evaluated to one loop order. The renormalization group flow also determines the phase diagram of the system, and we present a detailed analysis of the spinodal limiting the region of local stability of the homogeneous solution. Even in tree approximation the renormalized theory yields results markedly different from both Flory-Huggins theory or simple scaling considerations.
\end{abstract}

\section{Introduction.}

Physical properties of solutions containing two chemically different polymer species in a common solvent have been extensively studied both experimentally and theoretically. In particular, much effort [1] has aimed at the determination of the phase diagram, the experiments being usually interpreted within the frame work of a Flory-Huggins approach. Since phase separation often occurs at quite low concentrations, the validity of such an interpretation is doubtful, and this paper is mainly devoted to an analysis of the spinodal by means of the renormalization group [RG]. This method has been applied to ternary solutions only recently [3-5], all but the work of Joanny et al. [5] being restricted to an analysis of the second virial coefficient. In reference [5] the interpenetration function is calculated and the osmotic pressure in the semidilute limit is discussed with special regard to corrections to the excluded volume behaviour.

The work presented here in some respects parallels that of reference [5]. To give an adequate discussion of the spinodal, we, however, have to stress the nonlinear crossover behaviour of the coupling constant flow. Indeed we will find that not only the interpenetration function but also the spinodal is governed by the RG flow in all the accessible range of the coupling constants. Two properties of the RG flow are of special importance : first, as noted before [5], among the numereous fixed points found for this problem the globally stable one corresponds to the excluded volume limit where all renormalized coupling constants become equal to the same fixed point value $g^{*}$. Second, the renormalized coupling among different polymer species is found to be bounded from above which leads to the existence of a well defined limit of strong incompatibility. These two features of the RG flow give rise to phase diagrams which differ considerably both from the Flory-Huggins theory and from simple scaling results.

The organization of this article is as follows : in section 2 we calculate and analyse the RG flow. In section 3 the second virial coefficient and the 
interpenetration function are determined to one loop order, and the latter is analysed numerically for typical situations. In section 4 the spinodal is discussed. Being interested here in principle features, we restrict ourselves to the simplest (tree-) approximation and to a special type of «symmetric» solutions. Only the excluded volume limit and the $\theta$-limit are discussed in full generality. In section 5 we summarize our results and touch on the question of possible experimental verification.

\section{Renormalized coupling constants.}

We consider two monodisperse polymer species dissolved in a common solvent, which is a good or moderate solvent for each species separately. We denote by $c_{\mathrm{p}}^{(a)}$ or $N^{(a)}$ the polymer number concentration or chain length of the $a$-th species. The monomer size $l$ is taken to be the same for both species, an allowed simplification since a difference in $l$ can be absorbed into nonuniversal scale factors. The dimensionless unrenormalized coupling constant for $a-b$ contacts is denoted by $g_{a b, 0}=f_{a b, 0} g^{*}$, where $g^{*}$ is the usual fixed point coupling constant.

Renormalization is a way to analyse the influence of a change of the microscopic length scale $l \rightarrow l \lambda^{-1}$. Since this can be studied in the dilute limit the RG equations for the renormalized quantities $N_{\mathbf{R}}^{(a)}(\lambda)$, $c_{\mathrm{pR}}^{(a)}(\lambda), f_{a a}(\lambda)$ are not influenced by the presence of other polymer species and can be taken from previous work [6] $\left(^{1}\right)$.

$$
\begin{aligned}
\lambda & =\left(\frac{1-f_{a a}}{1-f_{a a, 0}}\right)^{1 / \omega} \cdot\left(\frac{f_{a a, 0}}{f_{a a}}\right)^{1 / \varepsilon} \\
N_{\mathrm{R}}^{(a)} & =\left(\frac{1-f_{a a}}{1-f_{a a, 0}}\right)^{1 / v \omega}\left(\frac{f_{a a, 0}}{f_{a a}}\right)^{2 / \varepsilon} S_{0, a} N^{(a)} \\
c_{\mathrm{pR}}^{(a)} & =\lambda^{-d} l^{d} c_{\mathrm{p}}^{(a)} .
\end{aligned}
$$

Here $d=4-\varepsilon$ is the space dimension, and $\omega$ and $v$ denote critical exponents. $S_{0, a}=S_{0}\left(f_{a a, 0}\right)$ is some microscopic scale factor.

The new feature of the present problem is the renormalization of $f_{12}$. A one-loop calculation yields the differential $\mathrm{RG}$ equation

$$
\frac{\mathrm{d} \ln f_{12}}{\mathrm{~d} \ln \lambda}=-\varepsilon+\frac{\varepsilon}{2} f_{12}+\frac{\varepsilon}{4}\left(f_{11}+f_{22}\right)+\mathcal{O}\left(\varepsilon^{2}\right)
$$

If both diagonal couplings take fixed point values $\left(\theta\right.$-point : $f_{a a}=0$, or excluded volume limit : $\left.f_{a a}=1\right)$ this equation can easily be integrated to yield

$$
\lambda=\left(\frac{f_{12}^{*}-f_{12}}{f_{12}^{*}-f_{12,0}}\right)^{1 / \omega_{12}}\left(\frac{f_{12,0}}{f_{12}}\right)^{1 / \tau \varepsilon},
$$

( $\left.{ }^{1}\right)$ We use the massless renormalization scheme of reference [7]. the values of $f_{12}^{*}, \omega_{12}$, and $\tau$ being collected in table I. In addition, equation (2.4) allows for a closed solution in two other cases :

$f_{11}=0 ; f_{22}=f$, arbitrary; or vice versa

$f_{12}=\frac{3}{2} f\left[1-\left(1-\frac{3}{2} \frac{f_{0}}{f_{12,0}}\right)\left(\frac{1-f}{1-f_{0}}\right)^{3 / 4}\right]^{-1}$

$f_{11} \equiv f_{22}=f$

$f_{12}=f\left[1-\left(1-\frac{f_{0}}{f_{12,0}}\right)\left(\frac{1-f}{1-f_{0}}\right)^{1 / 2}\right]^{-1}$.

Equivalent results derived in a somewhat different renormalization scheme are given in reference [5]. For a comparison of the correction to scaling exponents given in that reference to $\omega_{12}$ as given in table I, we note that the surface $S$ used there scales like $\lambda^{2}$.

We explicitly discuss the symmetric case (2.7). The flow diagram (see Fig. 1) in the $f_{12}-f$ plane exhibits four fixed points : $\mathbf{A}\left(f_{12}=f=0\right) ; \mathbf{B}\left(f_{12}=0\right.$, $f=1) ; \mathrm{C}\left(f_{12}=2, f=0\right) ; \mathrm{D}\left(f_{12}=f=1\right)$. Only the fixed point $\mathrm{D}$ is stable, which implies that under renormalization the difference between species 1 and 2 ultimately vanishes [5]. The lines $f_{12} \equiv 0$ connecting (A, B), $f \equiv 0$ connecting $(\mathrm{A}, \mathrm{C}), f \equiv 1$ connecting (B, D), or $f_{12} \equiv f$ connecting (A, D) are exact trajectories of the RG. The separatrix connecting $(C, D)$ to the present accuracy is found as

$$
f_{12}=f\left[1-(1-f)^{1 / 2}\right]^{-1} .
$$

In the neighbourhood of the separatrix connecting (A, D) the flow is unstable. Points starting slightly above or below this line will reach point $\mathrm{D}$ along singular trajectories $f_{12}-1 \sim \pm(1-f)^{1 / 2} \sim \pm$ $(1-f)^{\omega_{12}(\mathrm{D}) / \omega}$ from opposite directions. It is only for the separatrix $(A, D)$ itself that $D$ is approached in a regular fashion.

Flow lines like those shown in figure 1 can experimentally be realized by changing the chain lengths or the concentrations at fixed temperature. A temperature change influences $f_{0}$ and $f_{12,0}$, and therefore the system follows lines different from the $\mathrm{RG}$ flow. In discussing temperature effects we will restrict ourselves to the

Table I. - Values characterizing the $\mathrm{RG}$ flow of the coupling between different polymer species, calculated to one loop order. $f_{12}^{*}$ : nontrivial fixed point. $\omega_{12}$ or $-\tau \varepsilon$ : correction to scaling exponent for the nontrivial or trivial fixed points, respectively.

\begin{tabular}{l|c|c|c} 
Symmetric couplings & $f_{12}^{*}$ & $\omega_{12}$ & $\tau$ \\
\hline$f_{11}=f_{22}=0$ & 2 & $\varepsilon$ & 1 \\
$f_{a a}=0 ; f_{b b}=1$ & $\frac{3}{2}$ & $\frac{3}{4} \varepsilon$ & $\frac{3}{4}$ \\
$f_{11}=f_{22}=1$ & 1 & $\frac{1}{2} \varepsilon$ & $\frac{1}{2}$
\end{tabular}




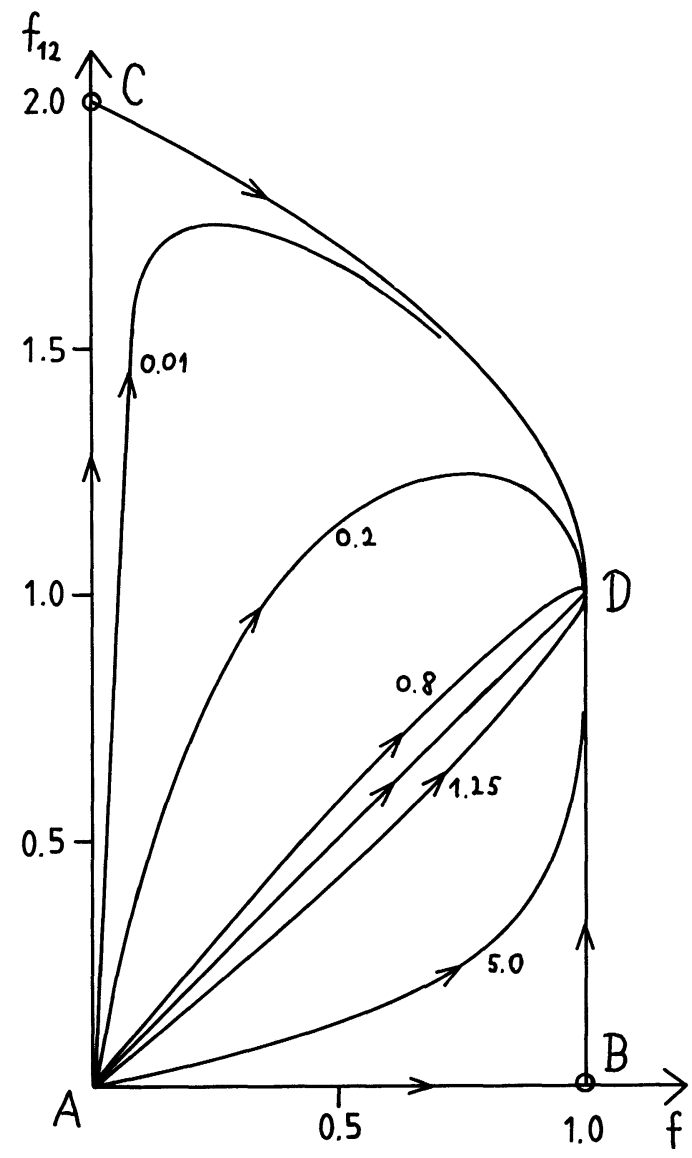

Fig. 1. $-f_{12}$ versus $f$ for a symmetric solution $f_{11}=f=f_{22}$. The flow lines depend on the parameter $f_{0} / f_{12,0}$, and the corresponding values are indicated in the figure.

«two-parameter» regime $f_{0} \ll 1$, taking $z=$ $f_{0}\left(S_{0} N\right)^{\varepsilon / 2}$ as a linear measure of $(T-\theta) / \theta$. $\left(N^{(1)}=N^{(2)}=N\right.$ in all cases analysed numerically).
$z_{12}=f_{12,0}\left(S_{0} N\right)^{\varepsilon / 2}$ is parametrized accordingly as

$$
z_{12}=A+B z
$$

The temperature variation of physical quantities is quite sensitive to the values of $A$ and $B$ as will be shown in the next sections.

In closing this section we note that all this discussion is by no means restricted to systems containing two monodisperse polymer species only. For more species, or polydisperse species, no new renormalizations occur and the results hold unchanged.

\section{Interpenetration ratio.}

Up to a constant of proportionality the interpenetration function $\psi^{(a b)}$ is defined as the ratio of the second virial coefficient $A_{2}^{(a b)}$ of a pair of chains divided by an appropriate combination of the radii of gyration $R_{\mathrm{g}}^{(a)}$.

$$
\psi^{(a b)}=\frac{1}{(4 \pi)^{d / 2}} A_{2}^{(a b)}\left(R_{\mathrm{g}}^{(a)} R_{\mathrm{g}}^{(b)}\right)^{-d / 2} .
$$

Here $A_{2}$ is determined from the virial expansion of the osmotic pressure $\pi$

$$
\frac{\pi}{k_{\mathrm{B}} T}=\sum_{a} c_{\mathrm{p}}^{(a)}+\frac{1}{2} \sum_{a, b} A_{2}^{(a b)} c_{\mathrm{p}}^{(a)} c_{\mathrm{p}}^{(b)}+\cdots
$$

In reference [5] the leading corrections to $\psi^{(a b)}$ near various fixed points have been evaluated to order $\varepsilon^{2}$, and a crossover function is given for the "symmetric" solution $f_{a a}=f_{b b}$. We therefore can be here very short, mainly evaluating the crossover functions to illustrate the typical behaviour reflecting the coupling constant flow.

Within our renormalization scheme, a one-loop calculation yields

$$
\begin{aligned}
A_{2}^{(a b)}=(l / \lambda)^{d} g^{*} f_{a b} N_{\mathrm{R}}^{(a)} N_{\mathrm{R}}^{(b)}\left\{1+\frac{\varepsilon}{8} f_{a b}\left[3-2 \gamma_{\mathrm{Eu}}+\right.\right. \\
\left.\left.+\frac{\left(N_{\mathrm{R}}^{(a)}+N_{\mathrm{R}}^{(b)}\right)^{2}}{N_{\mathrm{R}}^{(a)} N_{\mathrm{R}}^{(b)}} \ln \left(N_{\mathrm{R}}^{(a)}+N_{\mathrm{R}}^{(b)}\right)-2 \ln \left(N_{\mathrm{R}}^{(a)} N_{\mathrm{R}}^{(b)}\right)-\frac{N_{\mathrm{R}}^{(a)}}{N_{\mathrm{R}}^{(b)}} \ln N_{\mathrm{R}}^{(a)}-\frac{N_{\mathrm{R}}^{(b)}}{N_{\mathrm{R}}^{(a)}} \ln N_{\mathrm{R}}^{(b)}\right]+\mathcal{O}\left(\varepsilon^{2}\right)\right\} \\
R_{\mathbf{g}}^{(a)^{2}}=\frac{d}{3}(l / \lambda)^{2} N_{\mathbf{R}}^{(a)}\left[1-\frac{\varepsilon}{8} f_{a a}\left(\frac{37}{12}-\gamma_{\mathrm{Eu}}-\ln N_{\mathrm{R}}^{(a)}\right)+\mathcal{O}\left(\varepsilon^{2}\right)\right]
\end{aligned}
$$

where $\gamma_{\mathrm{Eu}}=0.577 \ldots$ denotes Euler's constant. To order $\varepsilon$, equation (3.3) is consistent with the result of reference [3]. Fixing the scale factor $\lambda$ by imposing the constraint $N_{\mathbf{R}}^{(1)} \cdot N_{\mathbf{R}}^{(2)}=1$ and introducing the notation

$$
N_{\mathrm{R}}^{(1)} \equiv N_{\mathrm{R}}^{(2)^{-1}}=y
$$

we find

$$
\begin{array}{r}
\psi^{(a b)}=\frac{g^{*}}{(4 \pi)^{d / 2}}\left(\frac{3}{d}\right)^{d / 2} f_{a b}\left\{1+\frac{\varepsilon}{8} f_{a b}\left[3-2 \gamma_{\mathrm{Eu}}+\left(y+\frac{1}{y}\right)^{2} \ln \left(y+\frac{1}{y}\right)-\left(y^{2}-\frac{1}{y^{2}}\right) \ln y\right]+\frac{\varepsilon}{8}\left(f_{a a}+f_{b b}\right) \times\right. \\
\\
\left.\times\left(\frac{37}{12}-\gamma_{\mathrm{Eu}}\right)-\frac{\varepsilon}{8}\left(f_{a a}-f_{b b}\right) \ln y\right\} .
\end{array}
$$


We note that the choice (3.5) is not adequate for discussing the limits $y \rightarrow 0$ or $y \rightarrow \infty$. Within a somewhat different approach these limits have been analysed in references $[3,5]$. At the fixed point $f_{a b}=f_{a a}=f_{b b}=1$ and for $y=1$ the interpenetration function takes a universal value [8]

$$
\psi^{*}=\left(\frac{3}{d}\right)^{d / 2} \frac{g^{*}}{(4 \pi)^{d / 2}}\left\{1+\frac{\varepsilon}{2}\left(\ln 2-\gamma_{\mathrm{Eu}}\right)+\frac{55}{48} \varepsilon+\mathcal{O}\left(\varepsilon^{2}\right)\right\}
$$

independent of the renormalization scheme. [For a comparison to previous calculations $[9,10]$ we note the value of $\left.g^{*}=(4 \pi)^{d / 2} \Gamma(d / 2) \varepsilon / 8\left(1+\frac{5}{32} \varepsilon+\mathcal{O}\left(\varepsilon^{2}\right)\right)\right]$.

By virtue of the prefactor $f_{a b}$ in $(3.6), \psi^{(12)} / \psi^{*}$, if plotted as a function of $\psi^{(a a)} / \psi^{*}$, will trace out curves qualitatively similar to the RG flow lines (Fig. 1), provided $f_{a a}$ and $f_{12}$ are changed by increasing the chain lengths at fixed temperature. Experimentally it may be easier to increase temperature with the chain lengths held fixed. We have evaluated our analytical results for this case, assuming a fully symmetric situation $f_{a a} \equiv f_{b b} \equiv f, y=1$. The temperature variation is described by the two-parameter scheme, explained above. Typical results are shown in figure 2 . In this figure, curve (0) represents the extreme limit of incompatibility : $A \rightarrow \infty$ in equation (2.9). In this limit $f_{12}$ for given $f$ reaches its maximum value, and therefore this curve gives an upper bound to $\psi^{(12)} / \psi^{*}$ for given $\psi$ (calculated here only to one loop order, of course.) It is the direct image of the separatrix connecting $(C, D)$ in figure 1 , and its very existence is a nontrivial consequence of RG theory. Line (6) is the only other RG trajectory in figure 2. It represents the trivial case $f_{12} \equiv f$, where the polymer species are indistinguishable. Lines (1) or (2) correspond to systems which are strongly incompatible for $T=\theta: A \gg 1$. For curve (1) the system stays incompatible for all $z(B>1)$ whereas for curve $(2)(B<1)$ it reaches the region of compatibility for finite $z$. Thus $\psi^{(12)} / \psi^{*}$ crosses line (6) and approaches the fixed point from below. Lines (3) or (4) show the corresponding behaviour for a system which near $z=0$ is only weakly incompatible. Finally curve (5) represents the limiting case $B=0$. Here the fixed point $\mathrm{D}\left(f_{12}=f=1\right)$ is not reached but the curve in a singular fashion approaches point $B$ $\left(f_{12}=0, f=1\right)$. Thus figure 2 demonstrates that even the special case of a symmetric solution shows a rich variety in the functional dependence $\psi^{(12)}(\psi)$.

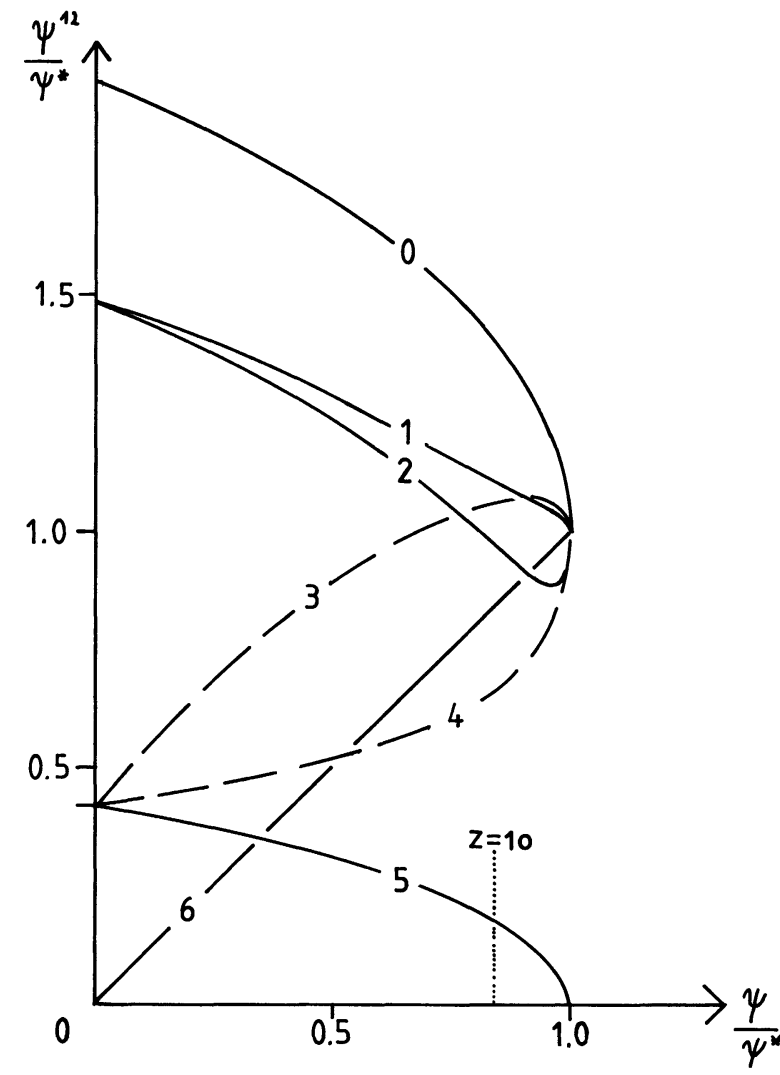

Fig. 2. $-\psi^{12} / \psi^{*}$ plotted as function of $\psi / \psi^{*}$ for a symmetric solution $f_{11}=f=f_{22}, N^{(1)}=N^{(2)}$. The interpenetration ratios are changed by changing the temperature. The different curves are explained in the text.

\section{The spinodal.}

4.1 Traditional apProach. - According to the Flory-Huggins theory the spinodal is determined by an equation of the structure [2]

$$
\begin{aligned}
{\left[1+\left(\varphi_{\mathrm{s}}^{-1}-2 \chi_{\mathrm{s} 1}\right) \varphi_{1} N^{(1)}\right]\left[1+\left(\varphi_{\mathrm{s}}^{-1}-2 \chi_{\mathrm{s} 2}\right) \varphi_{2} N^{(2)}\right] } & = \\
& =\left(\varphi_{\mathrm{s}}^{-1}-\chi_{\mathrm{s} 1}-\chi_{\mathrm{s} 2}+\chi_{12}\right)^{2} \varphi_{1} \varphi_{2} N^{(1)} N^{(2)}
\end{aligned}
$$

where $\varphi_{1}, \varphi_{2}$, or $\varphi_{\mathrm{s}}$ denote the volume fractions of the polymer species or the solvent, respectively. To reproduce the experimental results one has to allow for a dependence of the Flory-Huggins parameters $\chi_{s a}, \chi_{12}$ on temperature, concentrations, and chain lengths, and to explain these variations one needs new concepts going beyond Flory-Huggins theory. (In the related problem of the phase separation occurring 
below the $\theta$-temperature in a binary solution, empirically successful improvements of the Flory-Huggins theory have been presented. See, for instance, reference [12].) We here relate the difficulties of the traditional approach to the observation that for long chains the phase separation often occurs at low concentrations where Flory-Huggins theory cannot be trusted and where a renormalization group approach is appropriate.

Using concepts of scaling theory, De Gennes [13] has analysed the chain length dependence of $\chi_{12}$ for a symmetric system $\chi_{\mathrm{s} 1}=\chi_{\mathrm{s} 2}, N^{(1)}=N^{(2)}$. In the good solvent limit he predicts

$$
\chi_{12} \sim N^{v d-2} \chi_{12,0}^{v d-1} \stackrel{\approx}{\sim^{3}} N^{-0.24} \chi_{12,0}^{0.76}
$$

where $\chi_{12,0}$ is some « unrenormalized » Flory-Huggins parameter. In the $\theta$-region he finds

$$
\chi_{12} \sim N^{-1 / 2} \text {. }
$$

This prediction is claimed to be in good agreement with the experimental results of reference [11]. We will point out in the concluding section that our work raises some doubts in this interpretation.

4.2 LOWEST ORDER APPROXIMATION OF THE RENORMALIZED THEORY. - In the renormalized theory the spinodal obeys the equation

$$
\frac{\partial \mu_{\mathbf{R}}^{(1)}}{\partial c_{\mathbf{p}, \mathbf{R}}^{(1)}} \frac{\partial \mu_{\mathbf{R}}^{(2)}}{\partial c_{\mathrm{p}, \mathbf{R}}^{(2)}}-\frac{\partial \mu_{\mathbf{R}}^{(1)}}{\partial c_{\mathbf{p}, \mathbf{R}}^{(2)}} \frac{\partial \mu_{\mathbf{R}}^{(2)}}{\partial c_{\mathbf{p}, \mathbf{R}}^{(1)}}=0
$$

where $\mu_{\mathrm{R}}^{(a)}$ is the renormalized chemical potential of species $(a)$. In tree approximation $\mu_{\mathrm{R}}^{(a)}$ takes the form

$$
\mu_{\mathrm{R}}^{(a)}=\ln c_{\mathrm{p}, \mathrm{R}}^{(a)}+g^{*} \sum_{b} f_{a b} N_{\mathrm{R}}^{(a)} N_{\mathrm{R}}^{(b)} c_{\mathrm{p}, \mathrm{R}}^{(b)}
$$

If substituted into equation (4.4) this yields an equation of the same structure as the Flory-Huggins result.

$$
\left(1+g^{*} f_{11} c_{\mathrm{R}}^{(1)} N_{\mathrm{R}}^{(1)}\right)\left(1+g^{*} f_{22} c_{\mathrm{R}}^{(2)} N_{\mathrm{R}}^{(2)}\right)=\left(g^{*} f_{12}\right)^{2} c_{\mathrm{R}}^{(1)} c_{\mathrm{R}}^{(2)} N_{\mathrm{R}}^{(1)} N_{\mathrm{R}}^{(2)}
$$

We have here introduced the renormalized monomer concentration

$$
c_{\mathrm{R}}^{(a)}=c_{\mathbf{p}, \mathbf{R}}^{(a)} N_{\mathbf{R}}^{(a)} .
$$

Comparing equations (4.1) and (4.6) we can deduce expressions for the Flory-Huggins parameters in terms of renormalized quantities :

$$
\begin{gathered}
\left(\varphi_{\mathrm{s}}^{-1}-2 \chi_{\mathrm{s} a}\right) \varphi_{a} N^{(a)}=g^{*} f_{a a} c_{\mathrm{R}}^{(a)} N_{\mathrm{R}}^{(a)} \\
\left(\varphi_{\mathrm{s}}^{-1}-\chi_{\mathrm{s} 1}-\chi_{\mathrm{s} 2}+\chi_{12}\right)^{2} \varphi_{1} \varphi_{2} N^{(1)} N^{(2)}=\left(g^{*} f_{12}\right)^{2} c_{\mathrm{R}}^{(1)} c_{\mathrm{R}}^{(2)} N_{\mathrm{R}}^{(1)} N_{\mathrm{R}}^{(2)}
\end{gathered}
$$

The renormalized theory is valid only for small concentrations of the polymers where $\varphi_{\mathrm{s}}$ can be replaced by 1 . The volume fraction $\varphi_{a}$ is related to $c^{(a)}=c_{\mathrm{p}}^{(a)} N^{(a)}$ by a factor of the volume $v_{a} \approx l^{d}$ of a monomer, $\varphi_{a}=v_{a} c^{(a)}$, and $c^{(a)}$ can be expressed by renormalized quantities by virtue of equations $(2.1)-(2.3)$. This yields

$$
\begin{gathered}
\left(1-2 \chi_{\mathrm{s} a}\right) v_{a} l^{-d} S_{0, a}^{-2}=g^{*} f_{a a, 0}\left(\frac{1-f_{a a}}{1-f_{a a, 0}}\right)^{\frac{2-v d}{v \omega}} \\
\left(1-\chi_{\mathrm{s} 1}-\chi_{\mathrm{s} 2}+\chi_{12}\right) \frac{\sqrt{v_{1} v_{2}}}{l^{d} S_{01} S_{02}}=g^{*} f_{12} \sqrt{\frac{f_{11,0} f_{22,0}}{f_{11} f_{22}}} \cdot\left[\frac{\left(1-f_{11}\right)\left(1-f_{22}\right) .}{\left(1-f_{11,0}\right)\left(1-f_{22,0}\right)}\right]^{\frac{2-v d}{2 v \omega}} .
\end{gathered}
$$

We should stress that these relations hold only in the tree approximation of the renormalized theory and refer to $\chi$-parameters extracted from a determination of the spinodal. Other experiments may yield other effective parameters.

Since $(2-v d) / v \omega$ is positive, equation (4.9i) shows that a small value of $\left(1-2 \chi_{\mathrm{s} a}\right)$ does not necessarily imply that we are in the $\theta$-regime. It also vanishes in the excluded volume limit $f_{a a} \rightarrow 1$. The same holds true for $\chi_{12}$. In particular for a symmetric solution $f_{11}=f_{22}=f$ equation (4.9ii) reduces to

$$
\text { Const. } \chi_{12}=g^{*} f_{0}\left(\frac{f_{12}}{f}-1\right)\left(\frac{1-f}{1-f_{0}}\right)^{\frac{2-\nu d}{v \omega}} \text {. }
$$

Since $f_{12} / f-1 \sim(1-f)^{1 / 2}$ (compare Eq. $\left.(2.7)\right)$ for $f \rightarrow 1$ this vanishes faster than $1 / 2-\chi_{\mathrm{s}} \sim(1-f)^{(2-v d) / v \omega}$. This effect will render the simple scaling considerations invalid in the excluded volume regime. 
To proceed we need to fix the renormalized length scale $\lambda$. The choice of section 3 , viz $N_{\mathbf{R}}^{(1)}(\lambda) N_{\mathrm{R}}^{(2)}(\lambda)=1$, is not appropriate here since the spinodal will be seen to extend into the region of large overlap where $\lambda$ is to be determined by concentration rather than by chain length. In our previous work on binary solutions [6] we have fixed $\lambda$ to be of the order of the monomer density correlation length $\xi$, a choice which both was successful empirically and could be justified on theoretical grounds.

Searching for a generalization of this condition we here are faced with a matrix of density correlations

$$
I^{(a, b)}(q)=\left(c_{\mathrm{p}}^{(a)} c_{\mathrm{p}}^{(b)}\right)^{1 / 2} N^{(a)} N^{(b)} J_{a b}(q)
$$

replacing the single function $I(q)$ of reference [6]. In tree approximation we find the renormalized expression

$$
\left(J^{-1}\right)_{a b}=c_{\mathrm{p}, \mathrm{R}}^{(a) 1 / 2} N_{\mathrm{R}}^{(a)}\left(\frac{\delta_{a b}}{R_{a}\left(q^{2} l^{2} / \lambda^{2}\right)}+g^{*} f_{a b}\right) N_{\mathrm{R}}^{(b)} c_{\mathrm{p}, \mathrm{R}}^{(b) 1 / 2}
$$

where

$$
R_{a}(x)=\frac{2 c_{\mathrm{p}, \mathrm{R}}^{(a)}}{x^{2}}\left[\mathrm{e}^{-x} N_{\mathrm{R}}^{(a)}-1+x N_{\mathrm{R}}^{(a)}\right]
$$

is proportional to the Debye function. Diagonalizing $J^{-1}$ we can extract a correlation length from each of the two eigenvalues. One of these diverges at the spinodal and therefore corresponds to the critical mode of phase separation. The other length stays finite and is given by the lengthy expression

$$
\begin{aligned}
\frac{3}{d} \lambda^{-2}\left(\frac{\xi}{l}\right)^{2} & =\frac{N_{\mathrm{R}}^{(1)}+N_{\mathrm{R}}^{(2)}+\left(N_{\mathrm{R}}^{(1)}-N_{\mathrm{R}}^{(2)}\right)\left(f_{11} c_{\mathrm{R}}^{(1)} N_{\mathrm{R}}^{(1)}-f_{22} c_{\mathrm{R}}^{(2)} N_{\mathrm{R}}^{(2)}\right) Y^{-1 / 2}}{2+g^{*}\left(f_{11} c_{\mathrm{R}}^{(1)} N_{\mathrm{R}}^{(1)}+f_{22} c_{\mathrm{R}}^{(2)} N_{\mathrm{R}}^{(2)}+Y^{1 / 2}\right)} \\
Y & =\left(f_{11} c_{\mathrm{R}}^{(1)} N_{\mathrm{R}}^{(1)}-f_{22} c_{\mathrm{R}}^{(2)} N_{\mathrm{R}}^{(2)}\right)^{2}+4 f_{12}^{2} c_{\mathrm{R}}^{(1)} c_{\mathrm{R}}^{(2)} N_{\mathrm{R}}^{(1)} N_{\mathrm{R}}^{(2)}
\end{aligned}
$$

It is this length which tends to the density correlation length of the binary system if either the two polymer species become identical or one $c^{(a)}$ vanishes. Generalizing our work on binary systems we therefore impose the condition

$$
\frac{3}{d} \lambda^{-2}\left(\frac{\xi}{l}\right)^{2}=1
$$

Equations (4.14) (4.15), if combined with the RG equations (2.1) to (2.4) and the spinodal equation (4.6), provide a closed scheme to calculate the spinodal in renormalized tree approximation. The result clearly will not be quantitatively correct. We, however, expect it to give a good qualitative impression. Unfortunately, even for this simplest approximation, the system of equations has to be solved numerically. To show the principal features we restrict ourselves to a symmetric solution $N^{(1)}=N^{(2)}=N, f_{11,0}=f_{22,0}=f_{0}$. In addition we choose $c^{(1)}=c^{(2)}=c$, and we thus concentrate on the line of critical points where the spinodal surface and the surface of the first-order phase transition touch. As a result the equations simplify considerably. The spinodal is determined by

$$
g^{*} c_{\mathrm{R}} N_{\mathrm{R}}=\left(f_{12}-f\right)^{-1}
$$

and $\lambda$ implicitly is fixed by

$$
1=\frac{1}{N_{\mathrm{R}}}+\left(f+f_{12}\right) g^{*} c_{\mathrm{R}}
$$

$f_{12}$ being given by equation (2.7), of course. We will evaluate these equations in the domain of the twoparameter theory : $f_{0} \ll 1$, where simple scaling variables including temperature can be found.

Introducing the scaled concentration

$$
x=\left(S_{0} N\right)^{1-\varepsilon / 2} g^{*} S_{0} l^{d} c
$$

we can rewrite equations $(4.16)$ and (4.17) as

$$
\begin{aligned}
x= & z^{-1}\left(\frac{1}{\left(1-\frac{z}{z_{12}}\right)(1-f)^{1 / 2}}-1\right)(1-f)^{(v d-2) / v \omega} \\
& f^{2 / \varepsilon}(1-f)^{-\left(\frac{1}{2}+\frac{1}{v \omega}\right)}=\frac{1}{2} z^{2 / \varepsilon}\left(1-\frac{z}{z_{12}}\right)
\end{aligned}
$$

Thus the spinodal takes the scaling form

$$
x=x\left(z, z_{12}\right)
$$

and similar results hold for the Flory-Huggins parameters. Specifically, $\chi_{12}$ is found as

$$
\chi_{12}=\text { Const. } N^{-\varepsilon / 2} / x
$$

whereas $\chi_{\mathrm{s}}$ takes the scaling form

$$
\begin{aligned}
\frac{1}{2}-\chi_{\mathrm{s}}=\text { Const. } N^{-\varepsilon / 2} z(1 & -f)^{(2-v d) / v \omega}= \\
= & N^{-\varepsilon / 2} \tilde{\chi}_{s}\left(z, z_{12}\right) .
\end{aligned}
$$

We should stress that these scaling expressions hold only along the spinodal. 


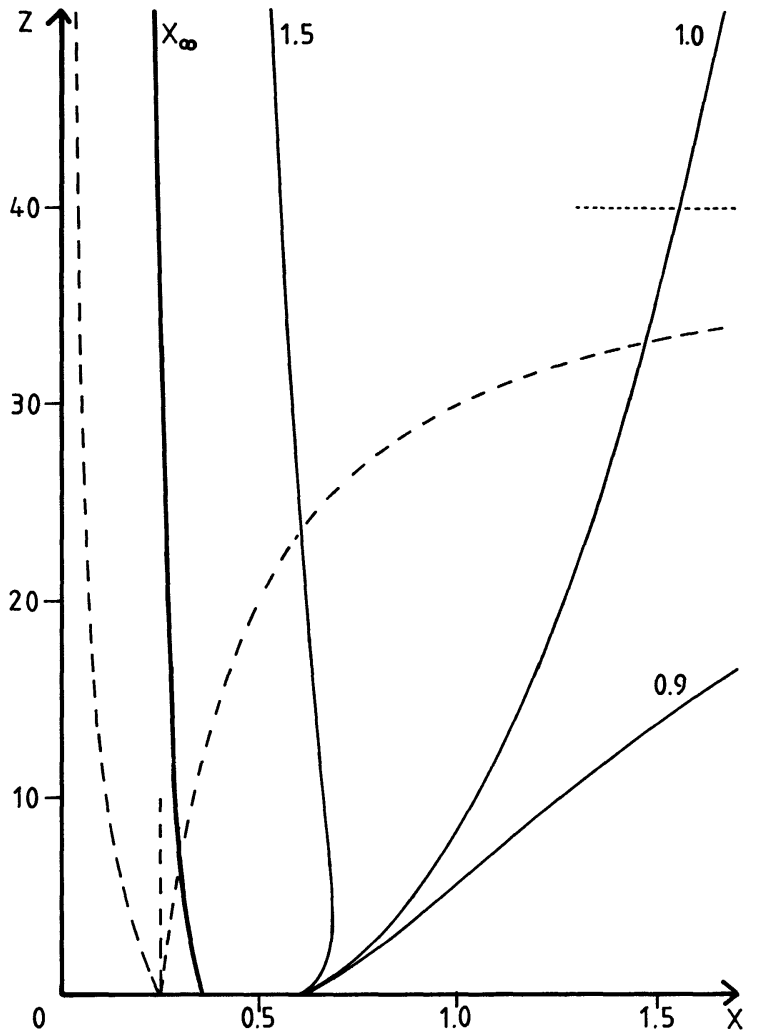

Fig. 3. - Spinodal curves $z(x)$ for values of the parameter $A=4.0$ and $B$ values $B=1.5,1.0,0.9$ as indicated. Broken lines represent the corresponding Flory-Huggins spinodals. The fat line represents the limit of strong incompatibility calculated according to the renormalization group. The dotted horizontal line gives the asymptote for $B=0.9$.

In figure 3 we have plotted the scaling form (4.20) of the spinodal for several values of the parameters $A, B$ in the parametrization (2.9) $: z_{12}=A+B z$, and in figure 4 we have shown the corresponding curves in the RG flow diagram $f_{12}$ versus $f$. In figure 3 we also included spinodal curves as calculated from the unrenormalized (Flory-Huggins) theory :

$$
x=\frac{1}{z_{12}-z} .
$$

The figures show typical examples illustrating the three different cases $B>1, B=1$, or $B<1$ distinguished by the behaviour in the excluded volume limit $z \gg 1(f \rightarrow 1)$, where equations (4.19), (4.22) reduce to

$$
\begin{gathered}
x=2^{\varepsilon / 2}\left(\frac{z}{2^{\varepsilon / 2}}\right)^{2 \rho / \varepsilon-1}\left(1-\frac{z}{z_{12}}\right)^{\rho-1} \\
\frac{1}{2}-\chi_{\mathrm{s}}=\text { Const. } N^{-\varepsilon / 2} z^{1-2 \sigma / \varepsilon}\left(1-\frac{z}{z_{12}}\right)^{-\sigma} \\
\rho=\frac{1-v d}{1+\frac{v \omega}{2}}+1 \stackrel{d=3}{\sim} 0.38
\end{gathered}
$$

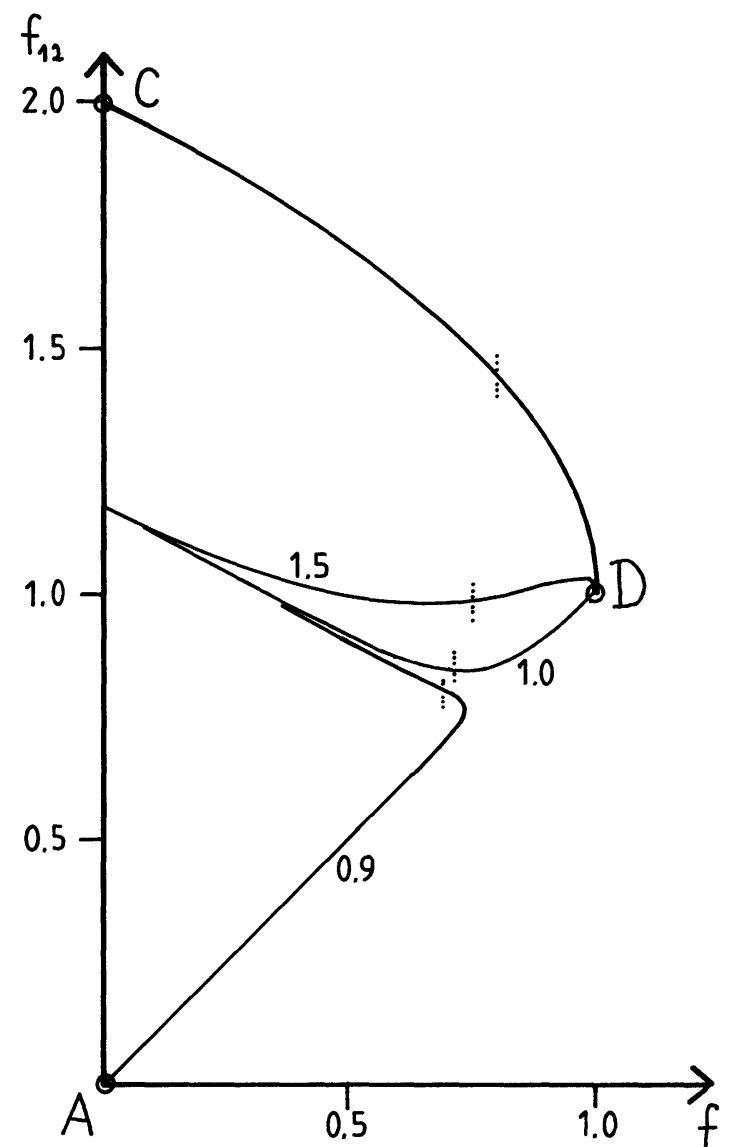

Fig. 4. - Flow lines $f_{12}$ versus $f$ corresponding to the renormalized spinodals of figure 3 . The dotted lines indicate the points $z \sim 10$.

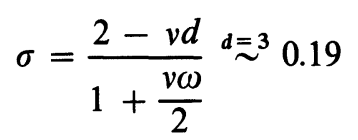

Taking $A>0$ always, we now discuss the three cases separately.

(i) $B>1$

In the asymptotic regime $B z \gg A$, equations (4.24) to (4.26) yield for $d=3$

$$
\begin{gathered}
x=1.2\left(1-\frac{1}{B}\right)^{-0.62} z^{-0.24} \\
\chi_{12} \sim f_{0}^{0.24} N^{-0.38} \\
\frac{1}{2}-\chi_{s} \sim f_{0}^{0.62} N^{-0.19}
\end{gathered}
$$

Equation (4.27) is to be compared to the unrenormalized result

$$
x=\frac{z^{-1}}{B-1} .
$$

For $B>1$, the spinodal asymptotically tends to $x=0$, the approach being much slower in renor- 
malized theory than in Flory-Huggins theory. In the $f_{12}-f$ diagram the spinodal approaches fixed point $D$ singularly from above

(ii) $B=1$

For $z \gg A$ we find

$$
\begin{aligned}
& x=1.2 A^{-0.62} z^{0.38} \quad \text { (renormalized) } \\
& x=\frac{1}{A} \quad \text { (unrenormalized) }
\end{aligned}
$$

and the $\chi$-parameters in the renormalized theory take the form

$$
\begin{aligned}
\chi_{12} & \sim f_{0}^{-0.38} N^{-0.69} \\
\frac{1}{2}-\chi_{\mathrm{s}} & \sim f_{0}^{0.81} N^{-0.10}
\end{aligned}
$$

In the $f_{12}-f$ diagram the spinodal approaches point $\mathrm{D}$ along the regular RG trajectory $f_{12}=f$.

(iii) $B<1$

In this case the factor $\left(1-\frac{z}{z_{12}}\right)$ becomes of dominant influence, its zero $z^{0}=A /(1-B)$ defining the temperature above which the polymers mix for all concentrations. In the renormalized theory this limit is approached as

$$
\begin{gathered}
x=1.2\left(z^{0}\right)^{-0.24}(1-B)^{-0.62}\left(1-\frac{z}{z^{0}}\right)^{-0.62} \\
\chi_{12} \sim N^{-0.38}\left(f_{0}^{0}\right)^{0.24}\left(1-\frac{f_{0}}{f_{0}^{0}}\right)^{0.62} \\
\frac{1}{2}-\chi_{s} \sim N^{-0.19}\left(f_{0}^{0}\right)^{0.62}\left(1-\frac{f_{0}}{f_{0}^{0}}\right)^{-0.19}
\end{gathered}
$$

The unrenormalized theory yields

$$
x=\frac{1}{1-B} \frac{1}{z_{0}-z} .
$$

In the $f_{12}-f$ diagram the spinodal may come close to the nontrivial fixed point $\mathrm{D}$ but finally turns around and approaches the trivial fixed point $\mathrm{A}$ along the separatrix $f_{12}=f$.

Independent of $B=$ Const. we may consider the limit of strong incompatibility $A \rightarrow \infty$. In this limit equations (4.19) yield a well defined curve $x=x_{\infty}(z)$ limiting a region $0 \leqslant x \leqslant x_{\infty}(z)$ where the two species are always compatible. In renormalized theory this region is of finite width, in contrast to the unrenormalized result $x \sim 1 / A \rightarrow 0$. In the excluded volume limit, equations (4.24)-(4.26) yield

$$
x_{\infty}(z)=1.2 z^{-0.24},
$$

$\chi_{12}$ or $\chi_{\mathrm{s}}$ being given by equations (4.28). In the RG flow diagram the spinodal coincides with the separatrix connecting points $\mathrm{C}$ and $\mathrm{D}$.
These considerations qualitatively explain the shape of the spinodal curves shown in figure 3 . It is obvious that renormalized theory and Flory-Huggins theory widely differ in their predictions. It seems appropriate to add a few words concerning the region where the limiting results (4.24), (4.25) hold. They are valid for $1-f \ll 1$, i.e.

$$
z^{2}\left(1-\frac{z}{z_{12}}\right) \gg 1
$$

For $B \geqslant 1$ this can be fulfilled for large enough $z$, and this is reflected by the fact that in that case the spinodals in the $f_{12}-f$ diagram end at fixed point $\mathrm{D}$. On the contrary for $B<1$ the spinodal does not reach point $\mathrm{D}$ but bends backwards towards the trivial fixed point. This is due to the screening of the effective interaction which occurs if the concentration increases $\left(c \sim N^{-1 / 2} x \rightarrow \infty\right.$ if $\left.z \rightarrow z_{0}\right)$. Thus equations (4.32), (4.33) have a region of validity only at intermediate $z$-values and only if $z_{0} \gg 1$. More precisely equation (4.36) leads to the condition

$$
A z_{0} \gg 1 \text {. }
$$

In the excluded volume limit we can easily extend our discussion to cover the concentration dependence of the spinodal in the symmetric case $f_{11,0}=f_{22,0}=f_{0}$, $N^{(1)}=N^{(2)}=N$, but $c^{(1)} \not \equiv c^{(2)}$. A general discussion of the excluded volume limit is presented in the next subsection. We therefore here only quote some results. In tree approximation we find

$$
\begin{aligned}
x^{(1)}+x^{(2)} & = \\
& =z^{2 / \varepsilon-1 / \rho}\left(2\left(1-\frac{z}{z_{12}}\right)\right)^{1-1 / \rho}\left(\frac{1}{x^{(1)}}+\frac{1}{x^{(2)}}\right)^{1 / \rho-1}
\end{aligned}
$$

where $x^{(a)}$ is defined by equation (4.18) with $c$ replaced by $c^{(a)}$. A simple reduced form of this result is found by extracting the critical concentration $x_{\text {cr }}$ (defined by $x^{(1)}=x^{(2)}=x_{\mathrm{cr}}$ ) which has been discussed extensively above.

$$
\frac{x^{(1)}+x^{(2)}}{2 x_{\mathrm{cr}}}=\left(\frac{1}{2} \frac{x_{\mathrm{cr}}}{x^{(1)}}+\frac{1}{2} \frac{x_{\mathrm{cr}}}{x^{(2)}}\right)^{1 / \rho-1}
$$

In the present approximation this is a universal form valid in the excluded volume limit. $x^{(a)} / x_{\mathrm{cr}}=c^{(a)} / c_{\mathrm{cr}}$ is independent of chain length or temperature. These parameters are absorbed in the critical concentration $c_{\mathrm{cr}}$. We should compare this to the Flory-Huggins result which takes the form

$$
\frac{c^{(1)}}{c_{\mathrm{cr}}}=\frac{\gamma+c^{(2)} / c_{\mathrm{cr}}}{(\gamma+2) c^{(2)} / c_{\mathrm{cr}}-1}
$$

depending on the parameter

$$
\gamma=\frac{\chi_{12}}{1-2 \chi_{s}} .
$$


Clearly expressions (4.39) or (4.40) give rise to quite different shapes of the spinodal surface. In particular equation (4.40) predicts that $c^{(a)} / c_{\mathrm{cr}}$ diverges at the finite value $c^{(b)} / c_{\mathrm{cr}}=(\gamma+2)^{-1}$, whereas in the renormalized theory the extreme wings of the surface approach $c^{(b)} / c_{\mathrm{cr}}=0$ :

$$
\frac{c^{(b)}}{c_{\mathrm{cr}}} \sim\left(\frac{c_{\mathrm{cr}}}{c^{(a)}}\right)^{\frac{\rho}{1-\rho}} \rightarrow 0 \quad \text { if } \quad \frac{c^{(a)}}{c_{\mathrm{cr}}} \rightarrow \infty
$$

At this point, however, we should recall that all our work holds only for dilute solutions $l^{d} c \ll 1$, so that these results are relevant only for very long chains $\left(c_{\mathrm{cr}} \sim N^{\rho-1} \rightarrow 0\right)$. In figure 5 we have plotted the universal curve (4.39), and we have compared it to the Flory-Huggins result with $\gamma=2 \rho /(i-2 \rho) \sim 3.2$ chosen such that both curves have the same curvature at the critical point.

We finally exhibit the concentration and chain length dependence of the Flory Huggins parameters. In terms of the reduced concentration $x^{(a)} / x_{\mathrm{cr}}$ we find

$$
\begin{aligned}
& 1-2 \chi_{\mathrm{s}}=\text { Const. } f_{0} z^{-2 \sigma / \varepsilon}\left(1-\frac{z}{z_{12}}\right)^{-\sigma}\left(\frac{x^{(1)}+x^{(2)}}{2 x_{\mathrm{cr}}}\right)^{\frac{2-v d}{v d-1}} \\
& \chi_{12}=\text { Const. } f_{0}\left(1-\frac{z}{z_{12}}\right)^{1-\rho} z^{-2 / \varepsilon \rho}\left(\frac{x^{(1)}+x^{(2)}}{2 x_{\mathrm{cr}}}\right)^{\frac{1+v \omega / 2}{v d-1}-1}
\end{aligned}
$$

consistent with our previous expressions. Since in the semidilute excluded volume limit $\lambda$, and thus $f$, depends only on a weighted mean of the concentrations, most simple expressions are found if equations (4.43) are rewritten in terms of $c^{(a)}$.

$$
\begin{aligned}
& 1-2 \chi_{\mathrm{s}}=\text { Const. } f_{0}^{2 / \varepsilon-\frac{2 / \varepsilon-1}{v d-1}}\left(l^{d} c^{(1)}+l^{d} c^{(2)}\right)^{\frac{2-v d}{v d-1}} \\
& \approx \text { Const. } f_{0}^{1.31}\left(l^{3} c^{(1)}+l^{3} c^{(2)}\right)^{0.31}, \quad d=3 \\
& \chi_{12}=\text { Const. }\left(1-\frac{f_{0}}{f_{12,0}}\right) f_{0}^{2 / \varepsilon-(2 / \varepsilon-1) \frac{1+v \omega / 2}{v d-1}}\left(l^{d} c^{(1)}+l^{d} c^{(2)}\right)^{\frac{1+v \omega / 2}{v d-1}-1} \\
& \approx \text { Const. }\left(1-\frac{f_{0}}{f_{12,0}}\right) f_{0}^{0.39}\left(l^{3} c^{(1)}+l^{3} c^{(2)}\right)^{0.61} \text {. }
\end{aligned}
$$

In tree approximation these parameters depend only on the total concentration and temperature.

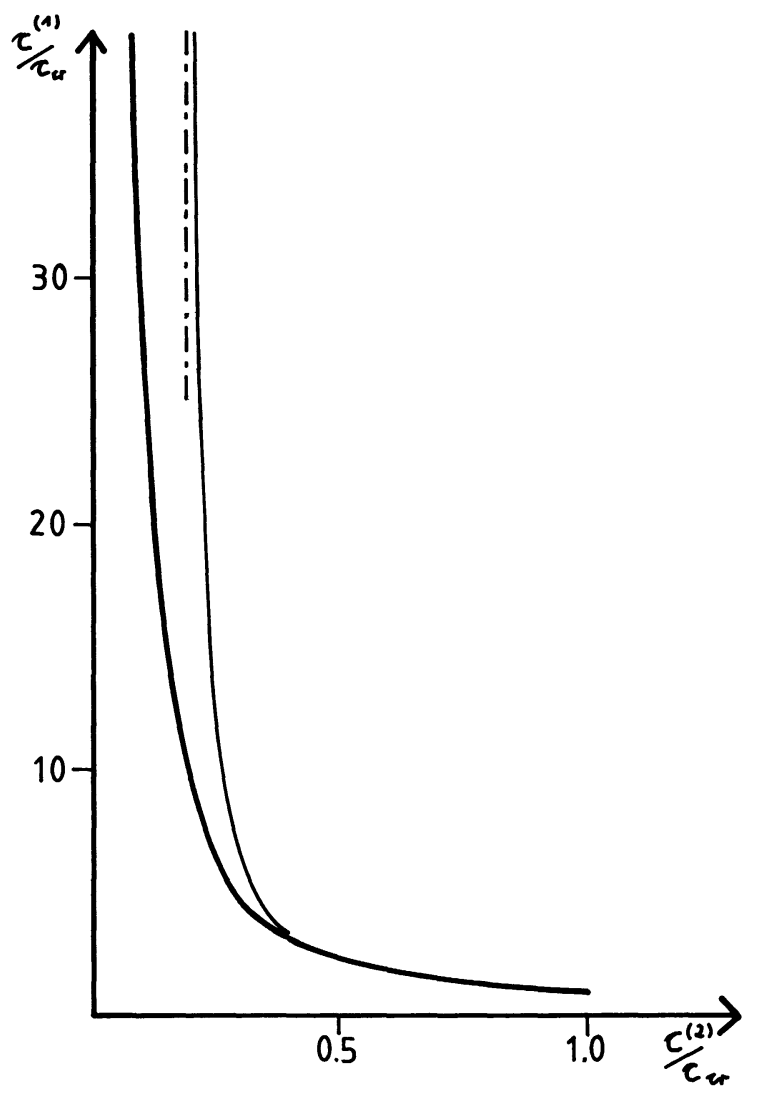

4.3 GENERAL DISCUSSION OF THE EXCLUDED VOLUME REGIME. - The excluded volume limit can be discussed analytically, the analysis not being restricted to tree approximation or to symmetric solutions. We start from equation (4.4) written in a form suggested by the tree approximation :

$$
\begin{aligned}
0=\frac{1}{g^{* 2} c_{\mathrm{R}}^{(1)} N_{\mathrm{R}}^{(1)} c_{\mathrm{R}}^{(2)} N_{\mathrm{R}}^{(2)}}+\frac{H(1,2)}{g^{*} c_{\mathrm{R}}^{(1)} N_{\mathrm{R}}^{(1)}}+ \\
+\frac{H(2,1)}{g^{*} c_{\mathrm{R}}^{(2)} N_{\mathrm{R}}^{(2)}}+G(1,2) .
\end{aligned}
$$

The functions $H$ and $G$ depend on the renormalized chain lengths, concentrations, and coupling constants. Inspection of the RG flow shows that in the excluded volume limit the system is driven to a fixed point where it cannot differentiate among the two species.

$\leftarrow$ Fig. 5. - Concentration dependence of the spinodal surface in the excluded volume limit. Only the sector $c^{(1)} \geqslant c^{(2)}$ is given. The fat or thin lines represent renormalized or FloryHuggins theory, respectively. The asymptotic behaviour of the Flory-Huggins theory is indicated by the broken line. 
At the fixed point no phase transition can occur and the spinodal has to run away into the region of infinite overlap $c_{\mathrm{R}}^{(a)} N_{\mathrm{R}}^{(a) v d-1} \rightarrow \infty$. This strongly suggests that $G(1,2)$ vanishes at the fixed point whereas $H(1,2)$ stays finite, a behaviour which is found in tree approximation : $G_{12}=f_{11} \cdot f_{22}-f_{12}^{2}, H(1,2)=f_{22}$. It is the basic assumption of the following derivation that this identifies the fixed point behaviour of $H$ and $G$ correctly.

To proceed we expand $G(1,2)$ around the fixed point. The leading contribution is due to

$$
1-f_{12}=\alpha(T) \lambda^{\omega_{12}}+\mathcal{O}\left(\lambda^{2 \omega_{12}}, \lambda^{\omega}\right)
$$

whereas corrections $1-f_{a a} \sim \lambda^{\omega}, \omega \sim 2 \omega_{12}$ can be neglected (compare Fig. 1 !). We thus write

$$
G(1,2)=-\alpha \lambda^{\omega_{12}} G^{\prime}(1,2) .
$$

$\lambda$ is still fixed by equation (4.15) with $\xi$ evaluated in tree approximation. There is no need to consider higher order terms in fixing $\lambda$. This yields

$$
\begin{gathered}
\frac{g^{*}\left(c_{\mathrm{R}}^{(1)} y^{1 / 2}+c_{\mathrm{R}}^{(2)} y^{-1 / 2}\right)^{2}}{c_{\mathrm{R}}^{(1)} y+c_{\mathrm{R}}^{(2)} y^{-1}}=1 \\
y=N_{\mathrm{R}}^{(1)} / N_{\mathrm{R}}^{(2)}
\end{gathered}
$$

where we have used that for $f \rightarrow 1$ the large overlap limit is reached where terms of order $1 / N_{R}$ vanish. Using the RG mapping we find

$$
\lambda^{d-1 / v}=\frac{\left(\tilde{c}^{(1)} y^{1 / 2}+\tilde{c}^{(2)} y^{-1 / 2}\right)^{2}}{\tilde{c}^{(1)} y+\tilde{c}^{(2)} y^{-1}}
$$

where

$$
\tilde{c}^{(a)}=g^{*} S_{0, a} f_{a a, 0}^{1 / \varepsilon(2-1 / v)} c^{(a)} .
$$

By virtue of equation (4.49) we can express the variables $c_{R}^{(a)}$ in terms of $y$ and of

$$
w=\frac{c_{\mathrm{R}}^{(1)}}{c_{\mathrm{R}}^{(2)}}=\frac{\tilde{c}^{(1)}}{\tilde{c}^{(2)}} .
$$

Furthermore the functions $H, G^{\prime}$ should be well behaved in the semidilute excluded volume limit $N_{\mathrm{R}}^{(a)} \rightarrow \infty, y=$ Const. and after this limit has been taken, the variables $N_{\mathrm{R}}^{(a)}$ occur in $H, G^{\prime}$ only in the combination $y$. Finally we note that the first term on the r.h.s. of equation (4.46) is of order $1 / N_{R}$ compared to the other terms and thus drops out. With all these considerations equation (4.46) takes the form

$$
\begin{aligned}
0=\frac{H(w, y)}{g^{*} c_{\mathrm{R}}^{(1)}(w, y) \tilde{z}_{1}^{2 / \varepsilon}}+\frac{H\left(w^{-1}, y^{-1}\right)}{g^{*} c_{\mathrm{R}}^{(2)}(w, y) \tilde{z}_{2}^{2 / \varepsilon}}- \\
-\alpha \lambda^{\omega_{12}+1 / v} G^{\prime}(w, y) \\
\tilde{z}_{a}^{2 / \varepsilon}=N_{\mathrm{R}}^{(a)} \lambda^{-1 / v}=S_{0, a} f_{a a, 0}^{1 / \varepsilon(2-1 / v)} N^{(a)},
\end{aligned}
$$

and with the help of equation (4.51) our final result reads

$$
\begin{aligned}
& \alpha\left[\frac{\left(\tilde{c}^{(1)} y^{1 / 2}+\tilde{c}^{(2)} y^{-1 / 2}\right)^{2}}{\tilde{c}^{(1)} y+\tilde{c}^{(2)} y^{-1}}\right]^{v \omega_{12}+1} \\
& =\tilde{z}_{1}^{-2 / \varepsilon} \hat{H}(w, y)+\tilde{z}_{2}^{-2 / \varepsilon} \hat{H}\left(\frac{1}{w}, \frac{1}{y}\right)
\end{aligned}
$$

where $\hat{H}$ is a combination of $H, G^{\prime}$ and $c_{\mathrm{R}}^{(a)}$. Reducing this result to its essential content we may write

$$
\alpha\left(\tilde{c}^{(1)} \tilde{c}^{(2)}\right)^{1 / 2 \frac{v \omega_{12}+1}{v d-1}}=\left(\tilde{z}_{1} \tilde{z}_{2}\right)^{-1 / \varepsilon} \tilde{H}(w, y) .
$$

To check the consistency of the derivation we note that for $\tilde{z}_{1} \sim \widetilde{z}_{2} \rightarrow \infty$ we find $\tilde{c}^{(1)} \sim \tilde{c}^{(2)} \rightarrow 0$ so that the excluded volume limit is reached properly. Also, the overlap diverges as

$$
\tilde{c} N^{v d-1} \sim \tilde{z}^{2 / \varepsilon(v d-1) \frac{v \omega_{12}}{1+v \omega_{12}}}
$$

For fixed temperature ( $f_{a b, 0}=$ Const., $\alpha=$ Const.), fixed relative chain length $y$ and fixed concentration ratio $w$ equation (4.57) predicts the power law

$$
c \sim N^{-\frac{v d-1}{1+v \omega_{12}}}
$$

which decreases less rapidly than $c \sim\left(\chi_{12} N\right)^{-1} \sim$ $N^{1-v d}$ suggested by the scaling argument (compare Eq. (4.2)). It is easily seen that the scaling argument ignores the fact that at the fixed point the system is compatible so that $G(1,2) \rightarrow 0$. It thus misses the factor $\lambda^{\omega_{12}}$. In terms of equation $(4.10)$ for $\chi_{12}$ it takes care of the contribution $(1-f)^{(2-v d) / v \omega}$ but omits the factor $f_{12} / f-1 \sim(1-f)^{\omega_{12} / \omega}$. Comparison of equations (4.24) and (4.57) yields a scaling relation for the exponent $\rho$

$$
\rho-1=\frac{1-v d}{1+v \omega_{12}},
$$

which by virtue of $\omega_{12}=\frac{\omega}{2}+\mathcal{O}\left(\varepsilon^{2}\right)$ is consistent with equation (4.26i).

The corresponding result for $\sigma$ reads

$$
\sigma=\frac{2-v d}{1+v \omega_{12}}
$$

Of most interest is the variation of the spinodal with temperature. Unfortunately this cannot be extracted from (4.57) without knowledge of $\tilde{H}$ since all the variables $\tilde{c}, \tilde{z}, w, y, \alpha$ depend on temperature. A great simplification occurs in the symmetric case $f_{11,0}=$ $f_{22,0} \ll 1$, where $w$ and $y$ become independent of temperature and where we can easily estimate $\alpha$. Replacing in equation (2.7) the exponent $1 / 2$ by its exact value $\omega_{12} / \omega$ we find for $f_{12} \rightarrow 1$ :

$$
f_{12}-1=\left(1-\frac{f_{0}}{f_{12,0}}\right) f_{0}^{-\omega_{12} / \varepsilon} \lambda^{\omega_{12}}
$$


which yields

$$
\alpha=\left(1-\frac{f_{0}}{f_{12,0}}\right) f_{0}^{-\omega_{12 / \varepsilon}}
$$

It is now easily seen that with this form of $\alpha$ the discussion of the excluded volume limit given in the previous subsection remains valid in the symmetric case $f_{11,0}=f_{22,0}$ beyond tree approximation and for arbitrary $y$ or $w$.

4.4 The $\theta$-LIMIT. - In the $\theta$-limit $f_{11,0}=f_{22,0}=0$ the analysis of section 4.2 can easily be extended to cover the full dependence of the spinodal on concentrations and chain lengths. We, however, are still restricted to lowest order (tree-) approximation in $f_{12}$. The $R G$ equations $(2.1-2.3,2.5)$ reduce to

$$
\begin{gathered}
N_{\mathrm{R}}^{(a)}=\lambda^{2} S_{0, a} N^{(a)} \\
c_{\mathrm{R}}^{(a)}=\lambda^{2-d} l^{d} c^{(a)} S_{0, a} \\
\lambda=\left(\frac{2-f_{12}}{2-f_{12,0}}\right)^{1 / \omega_{12}^{\prime}}\left(\frac{f_{12,0}}{f_{12}}\right)^{1 / \varepsilon}
\end{gathered}
$$

where $\omega_{12}^{\prime}=\varepsilon+\mathcal{O}\left(\varepsilon^{2}\right)$ is the exponent corresponding to the fixed point $\mathrm{C}$ of figure 1 . Combining the equation for the spinodal

$$
1=g^{* 2} f_{12}^{2} c_{\mathrm{R}}^{(1)} c_{\mathrm{R}}^{(2)} N_{\mathrm{R}}^{(1)} N_{\mathrm{R}}^{(2)}
$$

with equation (4.15) :3/d $\lambda^{-2}(\xi / l)^{2}=1$, we find the condition

$4=N_{\mathrm{R}}^{(1)}+N_{\mathrm{R}}^{(2)}=\lambda^{2}\left(S_{0,1} N^{(1)}+S_{0,2} N^{(2)}\right)$.

Thus $\lambda$ along the spinodal is independent of concentration. As an immediate consequence the spinodal takes a universal form in terms of reduced variables $c_{\mathrm{R}}^{(a)} / c_{\mathrm{R}, \mathrm{cr}}^{(a)}=c^{(a)} / c_{\mathrm{cr}}^{(a)}$ :

$$
\frac{c^{(1)} c^{(2)}}{c_{\mathrm{cr}}^{(1)} c_{\mathrm{cr}}^{(2)}}=1
$$

The concentrations at the critical point are easily found to obey the relation

$$
c_{\mathrm{p}, \mathrm{cr}}^{(1)} \equiv c_{\mathrm{p}, \mathrm{cr}}^{(2)}=c_{\mathrm{p}, \mathrm{cr}} \text {. }
$$

Using this result together with equations (4.64), (4.65), (4.67), (4.68) we find an equation for $c_{\mathrm{p}, \mathrm{cr}}$ :

$$
g^{*} l^{d} c_{\mathrm{p}, \mathrm{cr}} \frac{S_{0,1} N^{(1)} S_{0,2} N^{(2)}}{\left(S_{0,1} N^{(1)}+S_{0,2} N^{(2)}\right)^{\varepsilon / 2}} 2^{\varepsilon}=f_{12}^{-1} .
$$

With $\omega_{12}^{\prime}=\varepsilon, f_{12,0} \ll 1$, equation $(4.66)$ can be solved analytically :

$$
\begin{gathered}
f_{12}=2 \frac{z_{12}}{1+z_{12}} \\
z_{12}=2^{-\varepsilon} f_{12,0}\left(S_{0,1} N^{(1)}+S_{0,2} N^{(2)}\right)^{\varepsilon / 2}
\end{gathered}
$$

which leads to the final result

$$
g^{*} l^{d} c_{\mathrm{p}, \mathrm{cr}}=\frac{2^{-\varepsilon}}{2}\left(1+\frac{1}{z_{12}}\right) \frac{\left(S_{0,1} N^{(1)}+S_{0,2} N^{(2)}\right)^{\varepsilon / 2}}{S_{0,1} N^{(1)} S_{0,2} N^{(2)}}
$$

A particularly simple expression is found for the Flory-Huggins parameter :

$$
\begin{aligned}
\chi_{12} & =\text { Const. } f_{12,0}\left(2-f_{12}\right)^{\varepsilon / \omega_{12}^{\prime}} \\
& =\text { Const. } \frac{f_{12,0}}{1+z_{12,0}}
\end{aligned}
$$

where in the last line we used the approximate value $\omega_{12}^{\prime}=\varepsilon$. We note that $\chi_{12}$ along the spinodal surface is independent of concentrations or the ratio of the chain lengths.

In the limit of weak incompatibility $z_{12} \ll 1$ equations (4.74), (4.75) reproduce Flory-Huggins theory. In the opposite limit $z_{12} \gg 1$ they are consistent with De Gennes' scaling result (4.3). Analysing this derivation we find that this scaling behaviour is a consequence of the existence of a fixed point (point $\mathrm{C}$ in Fig. 1) representing the strongly incompatible $\theta$-system. It therefore holds independent of tree approximation. The cross-over between the $\theta$-fixed point $A$ and fixed point $C$, as described by equations (4.74) or (4.75), will be subject to higher order corrections, of course.

\section{Summary and conclusions.}

In summary, we have presented an analysis of the RG flow for a solution containing several polymer species, and we have worked out the consequences of this flow for the interpenetration function (to one-loop order) and for the spinodal (in tree approximation). Whereas results on the interpenetration function have been recently published [5], the spinodal is treated here for the first time. New qualitative aspects arise from the fact that the renormalized coupling constant $f_{12}$, giving the interaction among different species, is bounded from above. This gives rise to the existence of a well defined limit of strong incompatibility. The interpenetration function $\psi^{(1,2)}$ is bounded from above, and in appropriately scaled variables $x \sim c N^{1-\varepsilon / 2}, \quad z \sim(T-\theta) N^{\varepsilon / 2}$ the spinodal does not collapse on the line $x \equiv 0$, even for infinitely strong incompatibility. Other important qualitative aspects result from the fact that the symmetric fixed point $f_{12}=f_{11}=f_{22}=1$ is the attractive one [5]. As a consequence the spinodal in the excluded volume limit tends to the region of infinite overlap and does not show the behaviour suggested by simple scaling ideas.

We believe that it should be possible to verify experimentally some part of the behaviour predicted here. It may be rather difficult to test the results for 
the interpenetration ratio. Determination of $\psi$ is notoriously difficult, and furthermore experiments in $\theta$-solvents cover only a small range in the $z$-variables (according to previous analysis [6] the range of $z$ in our normalization typically is restricted to $z \lesssim 10$ ). In good solvents one might be able to explore the interesting region near $\psi \sim \psi^{*}$.

We believe that our results for the spinodal could be checked more easily. Again experiments in good solvent and for a strongly incompatible system would be of most interest, giving rise to a determination of the exponent $\omega_{12}$ governing the excluded volume behaviour. In this context we want to comment on the experimental findings of reference [11] which have been taken as support [13] of the scaling law (4.3) valid in the strongly incompatible $\theta$-limit. From our analysis in section 4.4 we find that the experimentally observed concentration dependence of $\chi_{12}$ is at variance with this interpretation. Furthermore we know [6] that the binary subsystem polystyrenetoluene is in good solvent conditions. On the other hand our analysis of section 4.3 , assuming that the total system is close to the excluded volume limit, also does not apply : the observed chain length dependence of $\chi_{12}$ at the critical point seems to be too strong. We suppose that the second binary subsystem, viz. polyisobutylene-toluene, is in moderate solvent conditions, so that a calculation in the (unsymmetric) cross-over regime would be necessary. Unfortunately we have not been able to find data for this second subsystem which are of comparable quality to those available for polystyrene-toluene.

Being restricted to tree-approximation, our qualitative discussion of the spinodal can only be a first step. The one-loop corrections certainly have to be calculated. Also, to put the RG equation for $f_{12}$ on the same level as those for the $f_{a a}$, we should use a two-loop calculation similar to that given in reference [5]. Work in that direction is in progress. For a quantitative test of such calculations, experiments on ternary systems with well characterized binary subsystems would be most welcome.

\section{References}

[1] Krause, S., J. Macromol. Sci. Rev. Macromol. Chem. C 7 (1972) 251.

[2] Koningsveld, R., Chermin, H. A. G., Gordon, M., Proc. R. Soc. Lond. A 319 (1970) 331.

[3] Stepanow, S., Acta Polymerica 32 (1981) 98 ; Stepanow, S., Straube, E., Preprint, Merseburg (1985).

[4] Kosmas, M. K., J. Physique Lett. 45 (1984) L-889.

[5] Joanny, J.-F., Leibler, L., Ball, R., J. Chem. Phys. 81 (1984) 4640.

[6] SCHÄFER, L., Macromolecules 17 (1984) 1357, and references given therein.

[7] Brezin, E., Le Gillou, J. C., ZinN Justin, J., in Phase Transitions and Critical Phenomena, C.
Domb and M. S. Green Eds. (Academic Press, New York) 1976, Vol. 6, p. 125.

[8] Witten, T. A., SChäfer, L., J. Phys. A 11 (1978) 1843.

[9] Des Cloizeaux, J., J. Physique 42 (1981) 635.

[10] Douglas, J. F., Freed, K. F., Macromolecules 17 (1984) 1854.

[11] Van den Esker, M. W. J., VRiJ, A., J. Polymer Sci. Polym. Phys. Ed. 14 (1976) 1943.

[12] IRvine, P., GoRdon, M., Macromolecules 13 (1980) 761.

[13] De Gennes, P. G., Scaling Concepts in Polymer Physics, Sect. IV 4 (Cornell Univ. Press, Ithaca) 1979. 